\title{
Study of Reliable Control Via an Integral-Type Sliding Mode Control Scheme
}

\author{
Yew-Wen Liang, Member, IEEE, Li-Wei Ting, and Li-Gang Lin
}

\begin{abstract}
This paper studies the active reliable control issues for a class of second-order nonlinear uncertain systems using an integral-type sliding mode control (ISMC) strategy. The proposed ISMC reliable scheme is shown to be able to tolerate some of the actuators' faults whenever the fault detection and diagnosis information is available. The presented scheme also maintains the main advantages of the ISMC designs, including robustness, rapid response, and ease of implementation. When the uncertainties and the output of the faulty actuators are matched regarding the nominal healthy subsystem, the state trajectories of the nominal healthy subsystem and the uncertain faulty system are identical. As a result, the engineers may adopt an optimal strategy for the nominal system, creating a desired trajectory for that of the uncertain faulty system to follow. Simulation results demonstrate the benefits of the proposed scheme.
\end{abstract}

Index Terms-Integral-type sliding manifold, reliable control, sliding mode control (SMC), uncertain system.

\section{INTRODUCTION}

$\mathbf{R}$ ECENTLY, the study of reliable (or fault-tolerance) control, including fault detection and diagnosis (FDD) issues for performing the active reliable task, has attracted considerable attention (see, e.g., [1]-[19]). Since the repair and maintenance services are generally not able to be provided instantly, reliable control issues have become of paramount importance. The objective of reliable control is to design an appropriate controller such that the closed-loop system can tolerate the abnormal operations of specific control components and retain the overall system stability with acceptable system performance. Among the existing reliable control studies, some investigate the reliability issues for linear systems [17], [18], [20], and some investigate those for nonlinear systems [3], [10]-[13], [19]. The nonlinear reliability studies include the Hamilton-Jacobi (HJ)-based approach [10], [19] and the sliding mode control (SMC)-based approach [3], [11], [12]. Because the HJ-based approach is designed under an optimal strategy, its reliable controller is inevitably dependent on the solution of an associated HJ equation, which is, in general, difficult to solve. Although a power series method [21] may alleviate the difficulty through computer calculation, the obtained so-

Manuscript received January 7, 2010; revised July 30, 2010, January 17 , 2011, and April 28, 2011; accepted June 30, 2011. Date of publication July 18, 2011; date of current version March 30, 2012. This work was supported by the National Science Council, Taiwan, under Grants NSC 96-2221-E-009-228, NSC 97-2221-E-009-087, NSC 97-3114-E-009-002, NSC 98-2218-E-009-017, and NSC 99-2218-E-009-004.

The authors are with the Department of Electrical Engineering, National Chiao Tung University, Hsinchu 30010, Taiwan (e-mail: ywliang@cn. nctu.edu.tw; wayneteen@yahoo.com.tw; charles200183@yahoo.com.tw).

Color versions of one or more of the figures in this paper are available online at http://ieeexplore.iee.org.

Digital Object Identifier 10.1109/TIE.2011.2162211 lution is only an approximation, and the computational load grows quickly when the system is complicated. In contrast, the SMC reliable controllers do not require the solution of any HJ equation and retain the advantages of SMC designs, including rapid response, easy implementation, and robustness to model uncertainties and/or external disturbances [3], [22]-[26].

Although the SMC designs possess the aforementioned advantages, it was reported that the resulted closed-loop system might be sensitive to uncertainties and/or disturbances during the period of time in which the system state has not yet reached the sliding manifold [27]. To solve the reaching phase problem, an integral-type SMC (ISMC), which guarantees that the system trajectories will start in the manifold from the first time instant, and its applications have been studied recently (see, e.g., [3], [22]-[24], [28], and [29]). In addition to the absence of a reaching phase feature, the ISMC design also maintains the aforementioned advantages of SMC and the following three characteristics: First, the matched uncertainties and/or disturbances will be completely rejected whenever the system state remains on the sliding manifold; next, due to the fact that the maximum control magnitude of the SMC designs usually happens at the beginning of the reaching phase period, the maximum control magnitude required for ISMC is usually smaller than those of SMC designs; and, finally, the states of the nominal healthy subsystem and the matched-type uncertain system are exactly the same if the system state stays on the sliding manifold. The last feature provides us an extra degree of freedom to organize a suitable controller for the nominal system, creating a desired system state trajectory for the state of the uncertain system to follow. In light of the benefits mentioned earlier, this paper will investigate the reliability issues from the ISMC viewpoint. To the best of the authors' knowledge, this is the first such implementation that takes the advantages of the ISMC scheme at the reliable control issues. With this approach, the engineer is capable of addressing better system performance under different faulty situations.

The rest of this paper is organized as follows. Section II states the problem and the main goal of the study. It is followed by the design of the ISMC reliability laws. The analytical results are then applied in Section IV to the attitude control of a spacecraft. Finally, Section V provides the conclusions.

\section{Problem Statement}

Consider a class of $n$ second-order nonlinear control systems as described by

$$
\begin{aligned}
\dot{\mathbf{x}}_{1} & =\mathbf{x}_{2} \\
\text { and } \dot{\mathbf{x}}_{2} & =\mathbf{f}(\mathbf{x}, t)+G(\mathbf{x}, t) \mathbf{u}+\mathbf{d} .
\end{aligned}
$$


Here, $\mathbf{x}_{1}=\left(x_{1}, \ldots, x_{n}\right)^{T} \in \mathbb{R}^{n}, \mathbf{x}_{2}=\left(x_{n+1}, \ldots, x_{2 n}\right)^{T} \in$ $\mathbb{R}^{n}$, and $\mathbf{x}=\left(\mathbf{x}_{1}^{T}, \mathbf{x}_{2}^{T}\right)^{T}$ are the system states, $\mathbf{u}=\left(u_{1}, \ldots\right.$, $\left.u_{m}\right)^{T} \in \mathbb{R}^{m}$ are the control inputs, $\mathbf{d}=\left(d_{1}, \ldots, d_{n}\right)^{T} \in \mathbb{R}^{n}$ denote possible model uncertainties and/or external disturbances, $\mathbf{f}(\mathbf{x}, t) \in \mathbb{R}^{n}$ and $G(\mathbf{x}, t) \in \mathbb{R}^{n \times m}$ are smooth functions with $\mathbf{f}(\mathbf{0}, t)=\mathbf{0}$, and $(\cdot)^{T}$ denotes the transpose of a vector or a matrix.

In this study, we will investigate the active reliable control issues for Systems (1) and (2). That is, we assume that the actuator fault has been successfully detected and diagnosed by an FDD mechanism. The fault may be time varying and include degradation, amplification, and outage [11], [15]. Before the occurrence of faults, the engineers may take any kind of control strategy to fulfill their desired system performance. When the fault is detected and diagnosed, the control law is guided to switch to an active reliable law for ensuring system performance. Thus, after the fault is detected, we may divide the actuators into two groups, $\mathcal{H}$ and $\mathcal{F}$, within which we assume that all of the actuators in $\mathcal{H}$ are healthy while those in $\mathcal{F}$ experience faults. Moreover, we assume that the output values of the faulty actuators are successfully diagnosed by an FDD mechanism as

$$
\mathbf{u}_{\mathcal{F}}=\hat{\mathbf{u}}_{\mathcal{F}}+\Delta \mathbf{u}_{\mathcal{F}}
$$

where $\hat{\mathbf{u}}_{\mathcal{F}}$ and $\Delta \mathbf{u}_{\mathcal{F}}$ denote the estimated value and the estimation error, respectively. Let

$$
\begin{aligned}
G(\mathbf{x}, t) & =\left(G_{\mathcal{H}}(\mathbf{x}, t) \vdots G_{\mathcal{F}}(\mathbf{x}, t)\right) \\
\text { and } \mathbf{u} & =\left(\mathbf{u}_{\mathcal{H}}^{T} \vdots \mathbf{u}_{\mathcal{F}}^{T}\right)^{T} .
\end{aligned}
$$

Systems (1) and (2) can be

$$
\dot{\mathbf{x}}_{1}=\mathbf{x}_{2}
$$

and $\dot{\mathbf{x}}_{2}=\mathbf{f}(\mathbf{x}, t)+G_{\mathcal{H}}(\mathbf{x}, t) \mathbf{u}_{\mathcal{H}}+G_{\mathcal{F}}(\mathbf{x}, t)\left(\hat{\mathbf{u}}_{\mathcal{F}}+\Delta \mathbf{u}_{\mathcal{F}}\right)+\mathbf{d}$.

Here, we assume that $\mathbf{u}_{\mathcal{H}} \in \mathbb{R}^{k}, \hat{\mathbf{u}}_{\mathcal{F}}$ and $\Delta \mathbf{u}_{\mathcal{F}} \in \mathbb{R}^{m-k}$, and $m>k$. From [27], we know that

$$
I_{n}=G_{\mathcal{H}}(\mathbf{x}, t) G_{\mathcal{H}}^{+}(\mathbf{x}, t)+G_{\mathcal{H}}^{\perp}(\mathbf{x}, t) G_{\mathcal{H}}^{\perp+}(\mathbf{x}, t)
$$

where $I_{n} \in \mathbb{R}^{n \times n}$ denotes the identity matrix, $G_{\mathcal{H}}^{\perp}(\mathbf{x}, t) \in$ $\mathbb{R}^{k \times n}$ represents a matrix whose columns span the null space of $G_{\mathcal{H}}^{T}(\mathbf{x}, t)$, and $G_{\mathcal{H}}^{+}(\mathbf{x}, t)$ and $G_{\mathcal{H}}^{\perp+}(\mathbf{x}, t)$ denote the pseudoinverses of $G_{\mathcal{H}}(\mathbf{x}, t)$ and $G_{\mathcal{H}}^{\perp}(\mathbf{x}, t)$, respectively. Therefore, the uncertainties and/or disturbances, including the output of faulty actuators given in (7), can be projected into the column spaces of $G_{\mathcal{H}}(\mathbf{x}, t)$ and $G_{\mathcal{H}}^{\perp}(\mathbf{x}, t)$, in which the components of the projection are called the matched and the unmatched uncertainties regarding the healthy subsystem $\dot{\mathbf{x}}_{1}=\mathbf{x}_{2}$ and $\dot{\mathbf{x}}_{2}=\mathbf{f}(\mathbf{x}, t)+$ $G_{\mathcal{H}}(\mathbf{x}, t) \mathbf{u}_{\mathcal{H}}$, respectively [27]. However, due to the assumption that the estimated value $\hat{\mathbf{u}}_{\mathcal{F}}$ is available from the FDD mechanism, the matched part $G_{\mathcal{H}}(\mathbf{x}, t) G_{\mathcal{H}}^{+}(\mathbf{x}, t) G_{\mathcal{F}}(\mathbf{x}, t) \hat{\mathbf{u}}_{\mathcal{F}}$ of $G_{\mathcal{F}}(\mathbf{x}, t) \hat{\mathbf{u}}_{\mathcal{F}}$ in (7) can be directly compensated by the healthy controllers. Thus, the total uncertainties given in (7) reduce to

$$
\mathbf{d}_{t}:=G_{\mathcal{F}}(\mathbf{x}, t) \mathbf{u}_{\mathcal{F}}+\mathbf{d}-G_{\mathcal{H}}(\mathbf{x}, t) G_{\mathcal{H}}^{+}(\mathbf{x}, t) G_{\mathcal{F}}(\mathbf{x}, t) \hat{\mathbf{u}}_{\mathcal{F}} .
$$

According to (8), $\mathbf{d}_{t}$ can be further decomposed into matched and unmatched parts as shown in the following:

$$
\mathbf{d}_{t}=G_{\mathcal{H}}(\mathbf{x}, t) \mathbf{d}_{m}+\mathbf{d}_{u}
$$

where $\mathbf{d}_{m}=G_{\mathcal{H}}^{+}(\mathbf{x}, t) \mathbf{d}_{t}$ and $\mathbf{d}_{u}=G_{\mathcal{H}}^{\perp}(\mathbf{x}, t) G_{\mathcal{H}}^{\perp+}(\mathbf{x}, t) \mathbf{d}_{t}$. We impose the following four assumptions for succeeding analysis.

Assumption 1: Any $n$ columns taken from $G(\mathbf{x}, t)$ are linearly independent for any $(\mathrm{x}, t)$.

Assumption 2: When $k \leq n$, there exists a constant matrix $D_{\mathcal{H}} \in \mathbb{R}^{k \times n}$ such that $D_{\mathcal{H}} G_{\mathcal{H}}(\mathbf{x}, t)$ is uniformly invertible. When $k>n, D_{\mathcal{H}} \in \mathbb{R}^{n \times n}$ is chosen such that $D_{\mathcal{H}} G_{\mathcal{H}}(\mathbf{x}, t)$ has full rank.

Assumption 3: There exists a control $\mathbf{u}_{\mathcal{H} 0}$ such that the origin of the following nominal healthy subsystem

$$
\begin{aligned}
\dot{\mathbf{x}}_{1} & =\mathbf{x}_{2} \\
\text { and } \dot{\mathbf{x}}_{2} & =\mathbf{f}(\mathbf{x}, t)+G_{\mathcal{H}}(\mathbf{x}, t) \mathbf{u}_{\mathcal{H} 0}
\end{aligned}
$$

is uniformly asymptotically stable (UAS). That is, there exists a continuously differentiable function $V(\mathbf{x}, t)$ such that

$$
\begin{aligned}
& \gamma_{1}(\|\mathbf{x}\|) \leq V(\mathbf{x}, t) \leq \gamma_{2}(\|\mathbf{x}\|) \\
& \frac{\partial V(\mathbf{x}, t)}{\partial t}+\left(\frac{\partial V(\mathbf{x}, t)}{\partial \mathbf{x}}\right)^{T} \cdot\left(\begin{array}{c}
\mathbf{x}_{2} \\
\mathbf{f}(\mathbf{x}, t)+G_{\mathcal{H}}(\mathbf{x}, t) \mathbf{u}_{\mathcal{H} 0}
\end{array}\right) \\
& \quad \leq-\gamma_{3}(\|\mathbf{x}\|)
\end{aligned}
$$

where $\gamma_{1}, \gamma_{2}: \mathbb{R}^{+} \rightarrow \mathbb{R}^{+}$are class $\mathcal{K}_{\infty}$ functions and $\gamma_{3}$ is a class $\mathcal{K}$ function.

Assumption 4: There exist two nonnegative functions $\rho_{m}(\mathbf{x}, t)$ and $\rho_{u}(\mathbf{x}, t)$ such that

$$
\left\|\mathbf{d}_{m}\right\| \leq \rho_{m}(\mathbf{x}, t) \text { and }\left\|\mathbf{d}_{u}\right\| \leq \rho_{u}(\mathbf{x}, t)
$$

for all $\mathbf{x}$ and $t$, where $\mathbf{d}_{m}$ and $\mathbf{d}_{u}$ are given by (10).

Note that Assumption 1 claims that the system with any $n$ healthy actuators is controllable. Assumption 2 provides the condition so that the ISMC scheme can be successfully implemented. Assumption 3 asserts the performance of the healthy subsystem. Finally, Assumption 4 gives the upper bounds of possible matched and unmatched uncertainties for robustness analysis. From (10) and Assumption 4, it is clear that the more accurate the diagnosis of the FDD is, the smaller the function $\rho_{m}(\mathbf{x}, t)$ will be. The objective of this study is to organize an appropriate $\mathbf{u}_{\mathcal{H}}$ so that the origin of the closed-loop system is UAS under Assumptions 1-4.

\section{Reliable Controller Design}

To achieve the objective as stated earlier, in the following, we will employ the ISMC technique to perform the design task. Along the ISMC design procedure (see, e.g., [22] and [27]), we introduce the sliding manifold as the following:

$\mathbf{s}=\mathbf{s}(\mathbf{x}, t)$

$:=D_{\mathcal{H}} \cdot\left\{\mathbf{x}_{2}(t)-\mathbf{x}_{2}\left(t_{0}\right)-\int_{t_{0}}^{t}\left[\mathbf{f}(\mathbf{x}(\tau), \tau)+G_{\mathcal{H}}(\mathbf{x}(\tau), \tau) \mathbf{u}_{\mathcal{H} 0}\right] d \tau\right\}$. 
It follows from (6), (7), (10), and (16) that

$$
\begin{aligned}
\dot{\mathbf{s}}= & D_{\mathcal{H}} \cdot\left\{\dot{\mathbf{x}}_{2}(t)-\mathbf{f}(\mathbf{x}, t)-G_{\mathcal{H}}(\mathbf{x}, t) \mathbf{u}_{\mathcal{H} 0}\right\} \\
= & D_{\mathcal{H}} \cdot\left\{G_{\mathcal{H}}(\mathbf{x}, t) \mathbf{u}_{\mathcal{H}}+G_{\mathcal{F}}(\mathbf{x}, t) \mathbf{u}_{\mathcal{F}}+\mathbf{d}-G_{\mathcal{H}}(\mathbf{x}, t) \mathbf{u}_{\mathcal{H} 0}\right\} \\
= & D_{\mathcal{H}} G_{\mathcal{H}}(\mathbf{x}, t) \cdot\left\{\mathbf{u}_{\mathcal{H}}+G_{\mathcal{H}}^{+}(\mathbf{x}, t) G_{\mathcal{F}}(\mathbf{x}, t) \hat{\mathbf{u}}_{\mathcal{F}}+\mathbf{d}_{m}-\mathbf{u}_{\mathcal{H} 0}\right\} \\
& +D_{\mathcal{H}} \mathbf{d}_{u} .
\end{aligned}
$$

In order to keep the system state on the sliding manifold, we choose

$$
\mathbf{u}_{\mathcal{H}}= \begin{cases}\mathbf{u}_{\mathcal{H} 0}-G_{\mathcal{H}}^{+}(\mathbf{x}, t) G_{\mathcal{F}}(\mathbf{x}, t) \hat{\mathbf{u}}_{\mathcal{F}} & \text { if } \mathbf{s}=\mathbf{0} \\ \mathbf{u}_{\mathcal{H} 0}-G_{\mathcal{H}}^{+}(\mathbf{x}, t) G_{\mathcal{F}}(\mathbf{x}, t) \hat{\mathbf{u}}_{\mathcal{F}}-\mathbf{u}_{\mathcal{H} 1} & \text { if } \mathbf{s} \neq \mathbf{0}\end{cases}
$$

where

$$
\begin{gathered}
\mathbf{u}_{\mathcal{H} 1}=\rho(\mathbf{x}, t) \frac{\left[D_{\mathcal{H}} G_{\mathcal{H}}(\mathbf{x}, t)\right]^{T} \mathbf{s}}{\left\|\left[D_{\mathcal{H}} G_{\mathcal{H}}(\mathbf{x}, t)\right]^{T} \mathbf{s}\right\|} \\
\text { and } \rho(\mathbf{x}, t)>\rho_{m}(\mathbf{x}, t)+\left\|\left[D_{\mathcal{H}} G_{\mathcal{H}}(\mathbf{x}, t)\right]^{+} D_{\mathcal{H}}\right\| \cdot \rho_{u}(\mathbf{x}, t)
\end{gathered}
$$

Note that the reliable controller (18) involves the FDD information. Thus, we have the next result.

Theorem 1: Suppose that Systems (1) and (2) experience actuator faults at the control channels in $\mathcal{F}$ with estimated value $\hat{\mathbf{u}}_{\mathcal{F}}$ and error $\Delta \mathbf{u}_{\mathcal{F}}$ given by (3). Then, the origin of Systems (1) and (2) under Assumptions 1-4 and the control law given by (18)-(20) is UAS if

$$
\rho_{u}(\mathbf{x}, t) \cdot\left\|\left(\frac{\partial V(\mathbf{x}, t)}{\partial \mathbf{x}}\right)^{T}\left(\begin{array}{c}
\mathbf{0} \\
\Gamma(\mathbf{x}, t)
\end{array}\right)\right\|<\gamma_{3}(\|\mathbf{x}\|)
$$

for all $t$ and for all $\mathbf{x} \neq \mathbf{0}$, where

$$
\Gamma(\mathbf{x}, t):=I_{n}-G_{\mathcal{H}}(\mathbf{x}, t) \cdot\left[D_{\mathcal{H}} G_{\mathcal{H}}(\mathbf{x}, t)\right]^{+} \cdot D_{\mathcal{H}} \cdot
$$

Proof: From (17)-(20), Assumption 4, and the fact that $\left[D_{\mathcal{H}} G_{\mathcal{H}}(\mathbf{x}, t)\right] \cdot\left[D_{\mathcal{H}} G_{\mathcal{H}}(\mathbf{x}, t)\right]^{+}=I_{k} \quad$ for $\quad k \leq n \quad$ and $\left[D_{\mathcal{H}} G_{\mathcal{H}}(\mathbf{x}, t)\right] \cdot\left[D_{\mathcal{H}} G_{\mathcal{H}}(\mathbf{x}, t)\right]^{+}=I_{n}$ for $k>n$, we have

$$
\begin{aligned}
& \mathbf{s}^{T} \dot{\mathbf{s}}=\mathbf{s}^{T} D_{\mathcal{H}} G_{\mathcal{H}}(\mathbf{x}, t) \cdot\left\{-\rho(\mathbf{x}, t) \frac{\left[D_{\mathcal{H}} G_{\mathcal{H}}(\mathbf{x}, t)\right]^{T} \mathbf{s}}{\left\|\left[D_{\mathcal{H}} G_{\mathcal{H}}(\mathbf{x}, t)\right]^{T} \mathbf{s}\right\|}\right. \\
& \left.+\mathbf{d}_{m}+\left[D_{\mathcal{H}} G_{\mathcal{H}}(\mathbf{x}, t)\right]^{+} D_{\mathcal{H}} \mathbf{d}_{u}\right\} \\
& \leq\left\|\left[D_{\mathcal{H}} G_{\mathcal{H}}(\mathbf{x}, t)\right]^{T} \mathbf{s}\right\| \cdot\left\{-\rho(\mathbf{x}, t)+\rho_{m}(\mathbf{x}, t)\right. \\
& \left.+\left\|\left[D_{\mathcal{H}} G_{\mathcal{H}}(\mathbf{x}, t)\right]^{+} D_{\mathcal{H}}\right\| \cdot \rho_{u}(\mathbf{x}, t)\right\} \\
& <0
\end{aligned}
$$

for $\mathbf{s} \neq \mathbf{0}$. Since $\mathbf{s}\left(\mathbf{x}\left(t_{0}\right), t_{0}\right)=\mathbf{0}$, it follows from (23) that $\mathbf{s}(\mathbf{x}, t)=\mathbf{0}$ for all $t \geq t_{0}$, i.e., the system state remains on the sliding manifold for all $t \geq t_{0}$. To determine the sliding dynamics (motion equations on the sliding manifold), we use the equivalent control method (see, e.g., [26] and [27]). The equivalent control is obtained by solving the equation $\dot{\mathrm{s}}=\mathbf{0}$ from (17) as

$$
\begin{aligned}
\mathbf{u}_{\mathcal{H}}^{e q}=\mathbf{u}_{\mathcal{H} 0}-G_{\mathcal{H}}^{+}(\mathbf{x}, t) G_{\mathcal{F}} & (\mathbf{x}, t) \hat{\mathbf{u}}_{\mathcal{F}}-\mathbf{d}_{m} \\
& -\left[D_{\mathcal{H}} G_{\mathcal{H}}(\mathbf{x}, t)\right]^{+} D_{\mathcal{H}} \mathbf{d}_{u} .
\end{aligned}
$$

By substituting $\mathbf{u}_{\mathcal{H}}^{e q}$ into (6) and (7) and using (10), we have the sliding dynamics

$$
\begin{aligned}
\dot{\mathbf{x}}_{1} & =\mathbf{x}_{2} \\
\text { and } \dot{\mathbf{x}}_{2} & =\mathbf{f}(\mathbf{x}, t)+G_{\mathcal{H}}(\mathbf{x}, t) \mathbf{u}_{\mathcal{H} 0}+\Gamma(\mathbf{x}, t) \mathbf{d}_{u}
\end{aligned}
$$

where $\Gamma(\mathbf{x}, t)$ is given by (22). It then follows from (25) and Assumption 3 that

$$
\begin{aligned}
\dot{V}(\mathbf{x}, t)= & \frac{\partial V(\mathbf{x}, t)}{\partial t}+\left(\frac{\partial V(\mathbf{x}, t)}{\partial \mathbf{x}}\right)^{T} \\
& \cdot\left(\begin{array}{c}
\mathbf{f}(\mathbf{x}, t)+G_{\mathcal{H}}(\mathbf{x}, t) \mathbf{u}_{\mathcal{H} 0}+\Gamma(\mathbf{x}, t) \mathbf{d}_{u}
\end{array}\right) \\
\leq & -\gamma_{3}(\|\mathbf{x}\|)+\rho_{u}(\mathbf{x}, t) \cdot\left\|\left(\frac{\partial V(\mathbf{x}, t)}{\partial \mathbf{x}}\right)^{T}\left(\begin{array}{c}
\mathbf{0} \\
\Gamma(\mathbf{x}, t)
\end{array}\right)\right\| \\
< & 0
\end{aligned}
$$

for all $\mathbf{x} \neq \mathbf{0}$. Thus, the origin is UAS.

Remark 1: It is found from the proof of Theorem 1 that the matched uncertainties and/or disturbances can be completely rejected. When $k \geq n$ (i.e., the number of healthy actuators is greater than or equal to $n$ ), the range space of $G_{\mathcal{H}}(\mathbf{x}, t)$ is the whole $\mathbb{R}^{n}$ space, and hence, the uncertainties and the output of the faulty actuators are matched type, which can be completely rejected. Thus, the sliding dynamics given by (25) and (26) and that of the nominal healthy subsystem described in Assumption 3 are exactly the same. As a result, the state trajectories of the uncertain faulty system described by (6) and (7) and the nominal healthy subsystem are identical. Therefore, the engineer is allowed to organize an appropriate controller according to the system requirements for the nominal healthy subsystem, creating a desired system state trajectory for the state of the uncertain faulty system to follow.

Remark 2: Suppose that $k<n$ and $G(\mathbf{x}, t) \equiv G$ is a constant matrix. Then, under the settings of Theorem 1, the matched uncertainties (including the matched part of the output of the faulty actuators) regarding the healthy subsystem can be completely rejected, while the effect of the unmatched uncertainties in (25) and (26) remains minimum in the Euclidean norm sense if the matrix $D_{\mathcal{H}}$ in (16) is selected to be $D_{\mathcal{H}}=$ $G_{\mathcal{H}}^{+}$[27]. Under this setting, the control $\mathbf{u}_{\mathcal{H} 1}$ given by (19) is simplified to be

$$
\mathbf{u}_{\mathcal{H} 1}=\rho(\mathbf{x}, t) \frac{\mathbf{s}}{\|\mathbf{s}\|}
$$

Remark 3: It is known that the success of an active reliable scheme depends mainly on the performance of the supplementary FDD method, including detection speed and accuracy [2], [6], [7]. Therefore, it is important to select an FDD mechanism that is appropriate for an active mission according to system requirements. From the theoretical derivations presented earlier, it should be noted that the reliable scheme proposed in this study can tolerate the inaccuracy of FDD estimation provided that an upper bound of the estimation error is known. Thus, the proposed reliable scheme also provides engineers with the flexibility of choosing another well-developed FDD mechanism [2], [6], [7] in accordance with system requirements and FDD performances. 


\section{Application to Spacecraft ATtITUdE StABILIZATION}

An attitude model for a spacecraft along a circular orbit can be described in the same form as (1) and (2) with $n=3$ and $m=4$ [12]. The three Euler's angles $(\phi, \theta, \psi)$ and their derivatives are adopted as the six state variables. For simplicity, we assume in this study that the thruster is the only applied control force and there is actuator redundancy for the reliable task. Let $\mathbf{x}=\left(x_{1}, x_{2}, x_{3}, x_{4}, x_{5}, x_{6}\right)^{T}=(\phi, \theta, \psi, \dot{\phi}, \dot{\theta}, \dot{\psi})^{T}, \quad \mathbf{u}=$ $\left(u_{1}, u_{2}, u_{3}, u_{4}\right)^{T}$, and $\mathbf{f}(\mathbf{x}, t)=\left(f_{1}(\mathbf{x}, t), f_{2}(\mathbf{x}, t), f_{3}(\mathbf{x}, t)\right)^{T}$. The overall system dynamics has parameters as described in the following:

$$
\left.\begin{array}{rl}
f_{1}(\mathbf{x}, t)=\omega_{0} x_{6} c x_{3} c x_{2}-\omega_{0} x_{5} s x_{3} s x_{2}+\frac{I_{y}-I_{z}}{I_{x}} \\
\times\left[\begin{array}{l}
x_{5} x_{6}+\omega_{0} x_{5} c x_{1} s x_{3} s x_{2}+\omega_{0} x_{5} c x_{3} s x_{1} \\
+
\end{array}\right. \\
\quad+\omega_{0} x_{6} c x_{3} c x_{1}+\frac{1}{2} \omega_{0}^{2} s\left(2 x_{3}\right) c^{2} x_{1} s x_{2} \\
\quad+\frac{1}{2} w_{0}^{2} c^{2} x_{3} s\left(2 x_{1}\right)-\omega_{0} x_{6} s x_{3} s x_{2} s x_{1} \\
\quad-\frac{1}{2} \omega_{0}^{2} s^{2} x_{2} s^{2} x_{3} s\left(2 x_{1}\right)-\frac{1}{2} \omega_{0}^{2} s\left(2 x_{3}\right) s x_{2} s^{2} x_{1} \\
\left.\quad-\frac{3}{2} \omega_{0}^{2} c^{2} x_{2} s\left(2 x_{1}\right)\right]
\end{array}\right]
$$

Here, $I_{x}, I_{y}$, and $I_{z}$ denote the inertia with respect to the three body coordinate axes, $\omega_{0}$ denotes the constant orbital rate, and $c$ and $s$ denote the cosine and sine functions, respectively. Note that, Assumptions 1 and 2 are obviously satisfied since $G(\mathbf{x}, t)$ is a constant matrix, and a matrix that is composed of any three columns taken from $G$ is invertible. A candidate for $D_{\mathcal{H}}$ described in Assumption 2 is $D_{\mathcal{H}}=I_{3}$.
Moreover, the system is found to be controllable for any three of the control inputs being healthy; therefore, Assumption 3 is satisfied for $k \geq 3$, and we have an additional redundant control channel for the reliable control task. In this study, we assume that $I_{x}=I_{z}=2000 \mathrm{~kg} \cdot \mathrm{m}^{2}, I_{y}=400 \mathrm{~kg} \cdot \mathrm{m}^{2}$, and $\omega_{0}=1.0312 \times 10^{-3} \mathrm{rad} / \mathrm{s}$, and the angular positions are constrained to be $x_{1}, x_{3} \in[-\pi, \pi]$ and $x_{2} \in[-\pi / 2, \pi / 2]$.

For demonstration, we use the linear quadratic regulator (LQR) design [10] for the nominal controller $\mathbf{u}_{\mathcal{H} 0}$ of the ISMC scheme with $Q=2 I$ and $R=I$. Since the solution of the associated $\mathrm{HJ}$ equation for the LQR design is difficult to derive, we adopt the Taylor series expansion method [21] for $\mathbf{u}_{\mathcal{H}}$ up to order 3, which can be computed offline. The reliable scheme proposed in this paper can tolerate the FDD estimation error (see Remark 3). Therefore, in this study, we only adopt the observer and the observer parameters from [12, eqs. (10) and (11)]for demonstration of application because the adopted observer has been shown to be able to reflect the fault of any single actuator at an exponential rate and greatly attenuate high-frequency noises. It is also worth noting that any other well-developed FDD mechanism [4], [6] can be considered for application here. Before an alarm, all of the active reliable schemes adopt their conventional nonreliable designs as if all actuators are available. When there is an alarm, the associated active reliable controllers are activated according to the FDD information provided.

The numerical results of the study are summarized in Figs. 1-4. The four figures exhibit respectively the time histories of the six system states, the sliding variables $\mathbf{s}:=$ $\left(s_{1}, s_{2}, s_{3}\right)^{T}$, the four control inputs, and the residual and alarm signals. Among these figures, we consider the following four cases: the first two adopt the LQR reliable scheme [10] for the nominal system (labeled LQR1) and for the uncertain system (labeled LQR2), whereas the other two adopt the sliding mode reliable control scheme [12] (labeled SMRC) and the ISMC reliable scheme proposed in this paper (labeled ISMC). The parameters of the SMRC scheme are adopted from [12] as $M=2 I_{3}$ and $\eta=1$, while the parameter of the ISMC is $D_{\mathcal{H}}=$ $I_{3}$. In addition, we assume that $\mathbf{d}=0.08 \cdot(\sin (t), \cos (2 t)$, $\sin (3 t))^{T}+(0.1,0.1,0.1)^{T}, \quad \mathbf{x}(0)=(0.7,0.07,-1.5,-0.3$, $-1.3,0.2)^{T}$, and the upper bound $\rho_{m}(\mathbf{x}, t)$ in Assumption 4 is selected as $\left\|G_{\mathcal{H}}^{+}\right\| \cdot\|\mathbf{d}\|_{\infty}$, where $\|\mathbf{d}\|_{\infty}:=\sup _{t}\|\mathbf{d}\|$. To alleviate chatter, the sign function in SMRC is replaced by the saturation function with a boundary layer width of 0.02 , while the control (18) in ISMC is replaced by $\mathbf{u}_{\mathcal{H}}=\mathbf{u}_{\mathcal{H} 0}-G_{\mathcal{H}}^{+}(\mathbf{x}, t) G_{\mathcal{F}}(\mathbf{x}, t)$ $\hat{\mathbf{u}}_{\mathcal{F}}-\rho(\mathbf{x}, t)\left(\left[D_{\mathcal{H}} G_{\mathcal{H}}(\mathbf{x}, t)\right]^{T} \mathbf{s} / \epsilon(\mathbf{x}, \mathbf{s}, t)\right)$, where $\epsilon(\mathbf{x}, \mathbf{s}, t)=$ $\left\|\left[D_{\mathcal{H}} G_{\mathcal{H}}(\mathbf{x}, t)\right]^{T} \mathbf{s}\right\|$ if $\|\mathbf{s}\| \geq 0.02$ and $\epsilon(\mathbf{x}, \mathbf{s}, t)=0.02$ if $\|\mathbf{s}\|<0.02$. Finally, to demonstrate reliable performance, we assume that $u_{2}$ fails at $t=1 \mathrm{~s}$, and the threshold for the alarm is set as 0.05 , i.e., the alarm is triggered if the magnitude of any of the residual signals from the observer is greater than 0.05. It should be noted that the performance of an FDD mechanism is influenced by the presence of model uncertainties and/or disturbances, which might result in a false alarm. Therefore, the selection of the threshold is, in general, a tradeoff between the probability of a false alarm and the probability of missed detection [5]. Although the threshold can be set lower to promote the sensitivity of the FDD mechanism, a lower 


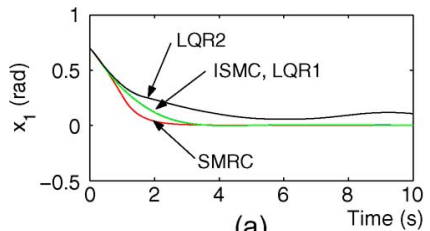

(a)

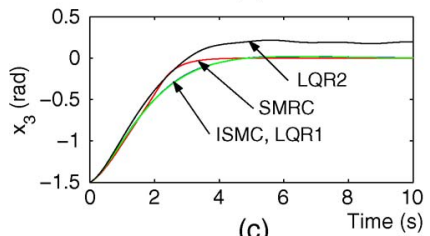

(c)

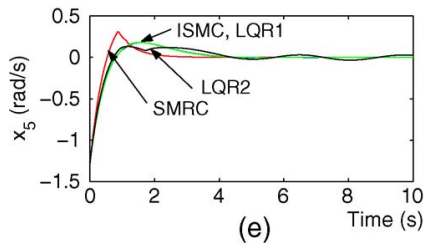

(e)

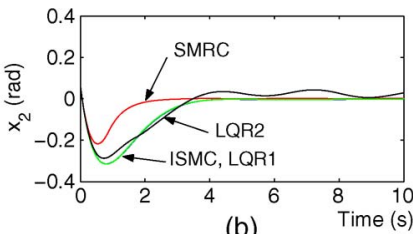

(b)

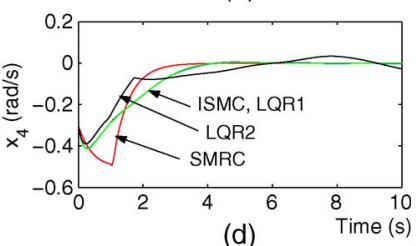

(d)

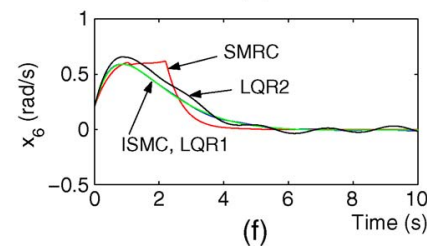

Fig. 1. Time history of the six system states with LQR, SMRC, and ISMC designs.

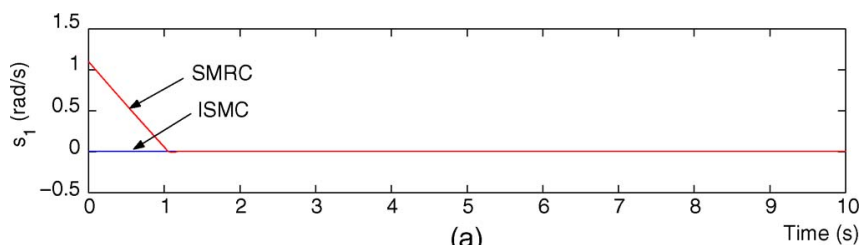

(a)
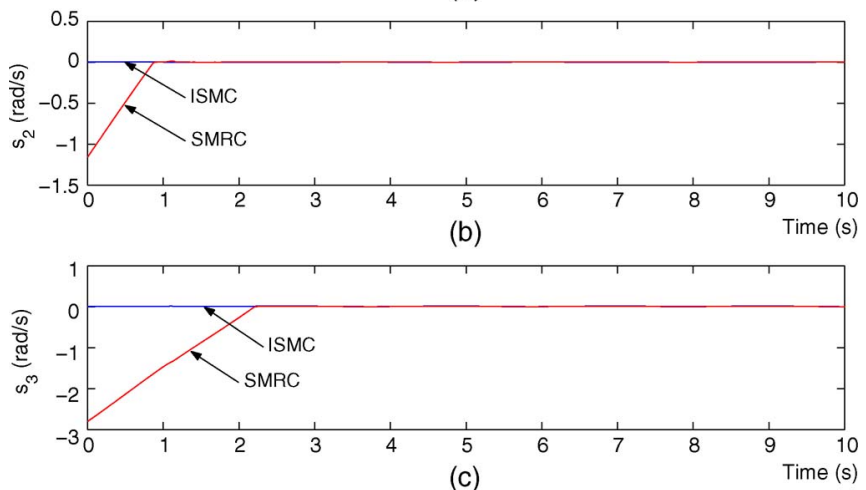

(c)

Fig. 2. Time history of the three sliding variables with SMRC and ISMC designs.

threshold setting might result in a false alarm for real and noisy applications [5], [16].

It is observed from Fig. 1 that the stabilization performance is, as expected, achieved for the SMRC and the ISMC designs. However, since the LQR is not a robust design, its system states are not found to be convergent to zero, particularly when there exist persistent uncertainties and/or disturbances. Moreover, the state trajectories of the ISMC and those for the nominal design LQR1 are found to be almost identical, which agrees with the theoretical conclusion. From Fig. 2, the sliding variables of the ISMC design are seen to be zero for all time. It implies that the system states remain on the sliding manifold for all $t$, which also agrees with the main results. In Fig. 4(a), the actuator fault is successfully detected by all of the three designs since the magnitude of the second residual signal exceeds the threshold near $t_{\mathrm{ISMC}} \approx 1.092 \mathrm{~s}$,

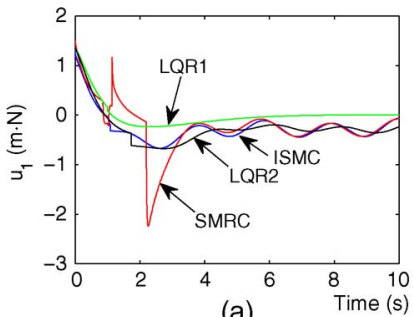

(a)

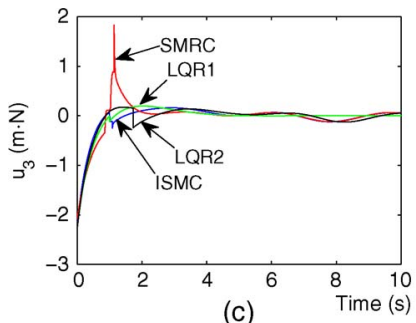

(c)

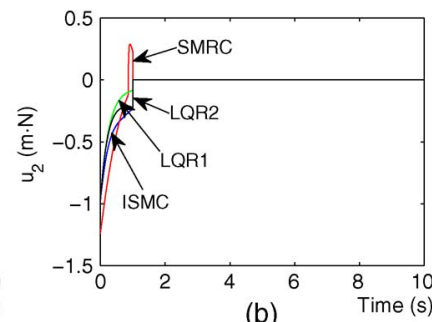

(b)

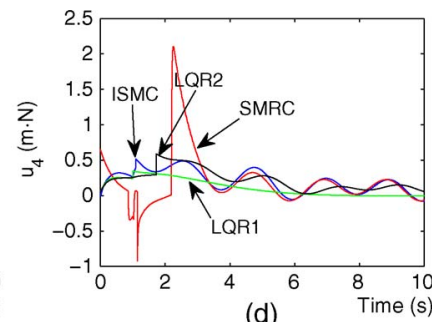

(d)
Fig. 3. Time history of the four control inputs with LQR, SMRC, and ISMC designs.

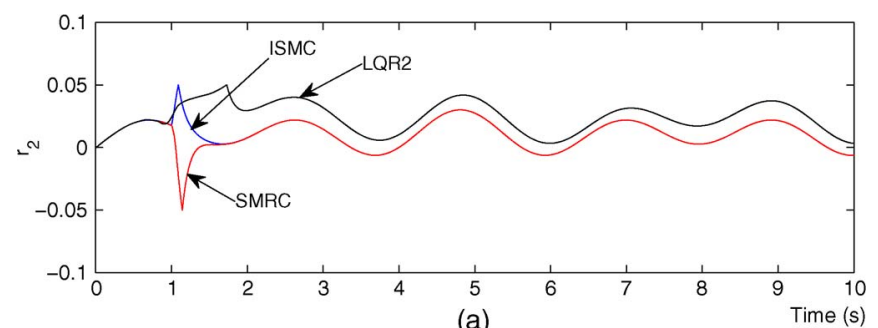

(a)

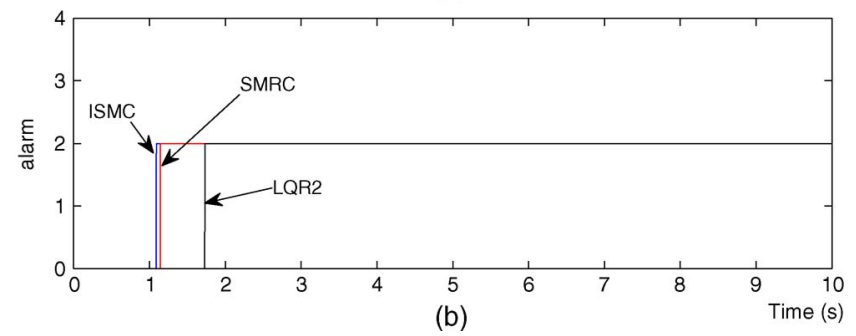

Fig. 4. Time history of (a) the second residual signals and (b) the alarm signals by the FDD observer with LQR, SMRC, and ISMC designs.

$t_{\mathrm{SMRC}} \approx 1.144 \mathrm{~s}$, and $t_{\mathrm{LQR} 2} \approx 1.73 \mathrm{~s}$. This can also be seen from the alarm signals given in Fig. 4(b) where the alarm value 2 denotes the fault of the second actuator. After the fault is successfully detected, the associated active reliable controllers are activated, and the magnitude of the residual signals soon decreases, as shown in Fig. 4(a). The persistent oscillation of the residual signal comes from the effect of the disturbance $\mathbf{d}$. It is also noted from Fig. 3 that there are several peaks for the control curves of the SMRC design. These peaks correspond to the system states reaching the sliding manifold and the switch of control due to the detection of fault, which can be seen from Figs. 2 and 4(b), where the time instants for the three sliding variables of SMRC reaching zero are $t_{s_{1}} \approx 1.17 \mathrm{~s}, t_{s_{2}} \approx 0.91 \mathrm{~s}$, and $t_{s_{3}} \approx 2.23 \mathrm{~s}$, respectively. Finally, since the ISMC design of this example adopts the LQR scheme for the nominal healthy subsystem, its performances are close to those of LQR1 except for the need of an extra control component to compensate for the uncertainties. In this example, the ISMC is found to have better performance than the SMRC in quadratic performance $\int \mathbf{x}^{T} Q \mathbf{x}+\mathbf{u}^{T} R \mathbf{u}$, energy 
consumption $\int \mathbf{u}^{T} \mathbf{u}$, and required maximum control magnitude $\|\mathbf{u}\|_{\infty}$ according to the following relations: $\left(\int \mathbf{x}^{T} Q \mathbf{x}+\right.$ $\left.\mathbf{u}^{T} R \mathbf{u}\right)_{\mathrm{LQR} 1}=10.24<\left(\int \mathbf{x}^{T} Q \mathbf{x}+\mathbf{u}^{T} R \mathbf{u}\right)_{\mathrm{ISMC}}=11.33<$ $\left(\int \mathbf{x}^{T} Q \mathbf{x}+\mathbf{u}^{T} R \mathbf{u}\right)_{\mathrm{SMRC}}=14.42, \quad\left(\int \mathbf{u}^{T} \mathbf{u}\right)_{\mathrm{LQR} 1}=2.24<$ $\left(\int \mathbf{u}^{T} \mathbf{u}\right)_{\mathrm{ISMC}}=3.35<\left(\int \mathbf{u}^{T} \mathbf{u}\right)_{\mathrm{SMRC}}=6.54$, and $\left(\|\mathbf{u}\|_{\infty}\right)_{\mathrm{LQR} 1}=$ $\left(\|\mathbf{u}\|_{\infty}\right)_{\text {ISMC }}=2.85<\left(\|\mathbf{u}\|_{\infty}\right)_{\text {SMRC }}=3.08$. Although the SMRC design consumes much more energy than the ISMC, it attains a larger convergence speed of the system states, which can be recognized in Fig. 1. From this simulation, it is concluded that the ISMC reliable design can not only achieve the stabilization performance when some of the actuators experience fault but also maintain the same state trajectory as the nominal healthy subsystem whenever the uncertainties are matched.

\section{CONCLUSION}

An ISMC stabilization scheme has been employed in this paper to study the active reliable control issues of a class of second-order nonlinear nonautonomous uncertain systems. Both matched and unmatched uncertainties were considered in this reliable design. Similar to those stated in [27], which were nonreliable designs and only investigated systems with constant control matrices, it was shown that the proposed reliable design can completely reject the matched uncertainty, while the effect of the unmatched uncertainty can also be made minimum through the setting of sliding manifold parameters under different faulty situations. As a result, the engineer is allowed to address better system performance for uncertain faulty system. It is worth noting that the matched uncertainty may become unmatched due to the actuators' outage. Therefore, for practical applications, it is important to have an effective FDD mechanism to efficiently realize the ISMC reliable control performances. An example and an observer were also given to demonstrate the benefits of the proposed reliable scheme. Nevertheless, the reliability study for more general systems and applications, including FDD development, needs further investigation.

\section{REFERENCES}

[1] B. Ayhan, H. Trussell, M. Chow, and M. Song, "On the use of a lower sampling rate for broken rotor bar detection with DTFT and AR-based spectrum methods," IEEE Trans. Ind. Electron., vol. 55, no. 3, pp. 14211434, Mar. 2008.

[2] M. Basseville, "On-board component fault detection and isolation using the statistical local approach," Automatica, vol. 34, no. 11, pp. 1391-1415, Nov. 1998.

[3] M. Corradini and G. Orlando, "Actuator failure identification and compensation through sliding modes," IEEE Trans. Control Syst. Technol., vol. 15, no. 1, pp. 184-190, Jan. 2007.

[4] C. De Angelo, G. Bossio, S. Giaccone, M. Valla, J. Solsona, and G. Garcia, "Online model-based stator-fault detection and identification in induction motors," IEEE Trans. Ind. Electron., vol. 56, no. 11, pp. 46714680, Nov. 2009.

[5] A. Emami-Naeini, M. Akhter, and S. Rock, "Effect of model uncertainty on failure detection: The threshold selector," IEEE Trans. Autom. Control, vol. 33, no. 12, pp. 1106-1115, Dec. 1988.

[6] I. Hwang, S. Kim, Y. Kim, and C. Seah, "A survey of fault detection, isolation, and reconfiguration methods," IEEE Trans. Control Syst. Technol., vol. 18, no. 3, pp. 636-653, May 2010.

[7] R. Isermann, R. Schwarz, and S. Stolzl, "Fault-tolerant drive-by-wire systems," IEEE Control Syst. Mag., vol. 22, no. 5, pp. 64-81, Oct. 2002.

[8] M. Khan and M. Rahman, "Development and implementation of a novel fault diagnostic and protection technique for IPM motor drives," IEEE Trans. Ind. Electron., vol. 56, no. 1, pp. 85-92, Jan. 2009.
[9] P. Lezana, J. Pou, T. Meynard, J. Rodriguez, S. Ceballos, and F. Richardeau, "Survey on fault operation on multilevel inverters," IEEE Trans. Ind. Electron., vol. 57, no. 7, pp. 2207-2218, Jul. 2010.

[10] Y.-W. Liang, D.-C. Liaw, and T.-C. Lee, "Reliable control of nonlinear systems," IEEE Trans. Autom. Control, vol. 45, no. 4, pp. 706-710, Apr. 2000.

[11] Y. Liang and S. Xu, "Reliable control of nonlinear systems via variable structure scheme," IEEE Trans. Autom. Control, vol. 51, no. 10, pp. 17211726 , Oct. 2006

[12] Y. Liang, S. Xu, and C. Tsai, "Study of VSC reliable designs with application to spacecraft attitude stabilization," IEEE Trans. Control Syst. Technol., vol. 15, no. 2, pp. 332-338, Mar. 2007.

[13] Y.-W. Liang, S.-D. Xu, and L.-W. Ting, "T-S model-based SMC reliable design for a class of nonlinear control systems," IEEE Trans. Ind. Electron., vol. 56, no. 9, pp. 3286-3295, Sep. 2009.

[14] O. Poncelas, J. Rosero, J. Cusido, J. Ortega, and L. Romeral, "Motor fault detection using a Rogowski sensor without an integrator," IEEE Trans. Ind. Electron., vol. 56, no. 10, pp. 4062-4070, Oct. 2009.

[15] R. Stengel, "Intelligent failure-tolerant control," IEEE Control Syst. Mag. vol. 11, no. 4, pp. 14-23, Jun. 1991.

[16] K. M. A. K. M. Tao and J. D. Lowrance, "Multiple-target tracking in dense noisy environments: A probabilistic mapping perspective," in Proc. SPIE, 2000, vol. 4048, pp. 474-485.

[17] R. Veillette, J. Medanic, and W. Perkins, "Design of reliable control systems," IEEE Trans. Autom. Control, vol. 37, no. 3, pp. 290-304, Mar. 1992.

[18] M. Vidyasagar and N. Viswanadham, "Reliable stabilization using a multi-controller configuration," Automatica, vol. 21, no. 5, pp. 599-602, 1985.

[19] G. Yang, J. Wang, and Y. Soh, "Reliable guaranteed cost control for uncertain nonlinear systems," IEEE Trans. Autom. Control, vol. 45, no. 11, pp. 2188-2192, Nov. 2000.

[20] F. Liao, J. Wang, and G. Yang, "Reliable robust flight tracking control: An LMI approach," IEEE Trans. Control Syst. Technol., vol. 10, no. 1, pp. 76-89, Jan. 2002.

[21] J. Huang and C. Lin, "Numerical approach to computing nonlinear $\mathrm{H}$ sub (infinity) control laws," J. Guid. Control Dyn., vol. 18, no. 5, pp. 989-996, 1995.

[22] W. Cao and J. Xu, "Nonlinear integral-type sliding surface for both matched and unmatched uncertain systems," IEEE Trans. Autom. Control, vol. 49, no. 8, pp. 1355-1360, Aug. 2004.

[23] M. Comanescu, L. Xu, and T. Batzel, "Decoupled current control of sensorless induction-motor drives by integral sliding mode," IEEE Trans. Ind. Electron., vol. 55, no. 11, pp. 3836-3845, Nov. 2008.

[24] M. Comanescu, "An induction-motor speed estimator based on integral sliding-mode current control," IEEE Trans. Ind. Electron., vol. 56, no. 9, pp. 3414-3423, Sep. 2009.

[25] R. DeCarlo, S. Zak, and G. Matthews, "Variable structure control of nonlinear multivariable systems: A tutorial," Proc. IEEE, vol. 76, no. 3 , pp. 212-232, Mar. 1988.

[26] V. I. Utkin, Sliding Modes in Control and Optimization. Berlin, Germany: Springer-Verlag, 1992.

[27] F. Castaños and L. Fridman, "Analysis and design of integral sliding manifolds for systems with unmatched perturbations," IEEE Trans. Autom. Control, vol. 51, no. 5, pp. 853-858, May 2006.

[28] H. Huerta, A. Loukianov, and J. Canedo, "Multimachine power-system control: Integral-SM approach," IEEE Trans. Ind. Electron., vol. 56, no. 6 , pp. 2229-2236, Jun. 2009.

[29] J. Xu and K. Abidi, "Discrete-time output integral sliding-mode control for a piezomotor-driven linear motion stage," IEEE Trans. Ind. Electron., vol. 55, no. 11, pp. 3917-3926, Nov. 2008.

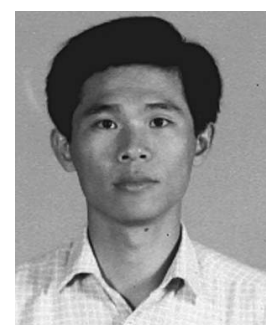

Yew-Wen Liang (M'02) was born in Taiwan in 1960. He received the B.S. degree in mathematics from the Tung Hai University, Taichung, Taiwan, in 1982, and the M.S. degree in applied mathematics and the Ph.D. degree in electrical and control engineering from the National Chiao Tung University, Hsinchu, Taiwan, in 1984 and 1998, respectively.

Since August 1987, he has been with the National Chiao Tung University, where he is currently an Associate Professor of electrical and control engineering. His research interests include nonlinear control systems, reliable control, and fault detection and diagnosis issues. 


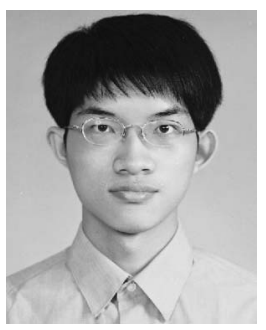

Li-Wei Ting received the M.S. degree in electrical engineering and control engineering from National Chiao Tung University, Hsinchu, Taiwan, in 2009.

His research interests include control engineering, fuzzy systems, and industrial electronics.

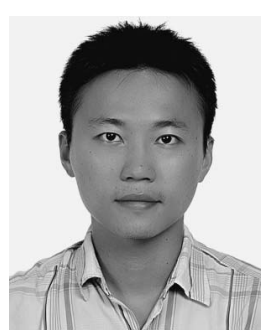

Li-Gang Lin received the B.S. and M.S. degrees in electrical control engineering from National Chiao Tung University, Hsinchu, Taiwan, in 2008 and in 2010 , respectively, where he is currently working toward the Ph.D. degree.

His research interests include nonlinear control systems, reliable control, and state-dependent Riccati equation. 